\title{
Characteristics of Lung Microbiota in Children's Refractory Mycoplasma pneumoniae Pneumonia Coinfected with Human Adenovirus B
}

\author{
Wenxiang Zhou, ${ }^{1}$ Jinglong Chen, ${ }^{2}$ Zhimin Xi, ${ }^{2}$ Yanyan Shi, ${ }^{2}$ Libo Wang, ${ }^{2}$ and Aizhen Lu ${ }^{2}$ \\ ${ }^{1}$ Division of Internal Medicine, Children's Hospital of Xiamen, Xiamen, Fujian, China \\ ${ }^{2}$ Division of Pulmonary Medicine, Children's Hospital of Fudan University, Shanghai, China
}

Correspondence should be addressed to Aizhen Lu; zal2008@163.com

Received 21 September 2021; Revised 7 December 2021; Accepted 31 December 2021; Published 17 January 2022

Academic Editor: Louis Detolla

Copyright (c) 2022 Wenxiang Zhou et al. This is an open access article distributed under the Creative Commons Attribution License, which permits unrestricted use, distribution, and reproduction in any medium, provided the original work is properly cited.

\begin{abstract}
Background. Both M. pneumoniae and human adenovirus (HAdV) are common causative agents of lower respiratory tract infection in children; nonetheless, the lung microbiota in patients with coinfection of HAdV and M. pneumoniae remain unexplored. Methods. Thirty-two children, diagnosed with refractory M. pneumoniae pneumonia (RMPP), entered into the oneyear study from July 1, 2019 to June 30, 2020. Among them, twenty-one entered into the M. pneumoniae monoinfection (MP) group and eleven entered into the M. pneumoniae and HAdV coinfection (MP\&ADV) group. The characteristics of the clinical findings were examined, and the lung microbiota was analyzed by metagenomic next generation sequencing (mNGS). Results. Eleven patients in the MP\&ADV group were coinfected with human mastadenovirus species B. The fever days lasted for significantly longer periods in the MP\&ADV group than in the MP group $(P<0.05)$. The percentage of $\mathrm{CD} 16^{+} \mathrm{CD} 56^{+}$cells was significantly higher in the MP\&ADV group than that in the MP group $(P<0.05)$. There were no significant differences in $\alpha$-diversity between the MP and MP\&ADV groups, but the $\beta$-diversity was clearly higher in the MP\&ADV group than that in the MP group $(P<0.05)$. At the microbial level, the top phylum of the MP BALF microbiota was Tenericutes; in contrast, it was Preplasmiviricota in the MP\&ADV BALF. There were significant differences in the relative abundance of Tenericutes and Preplasmiviricota between the two groups $(P<0.001)$. There was a strong positive correlation between human mastadenovirus $B$ and fever days, M. pneumoniae and level of IgA, and a strong negative correlation between Mycoplasma pneumoniae and PCT. Conclusions. In RMPP, the BALF microbiota in children with mono $M$. pneumoniae infection was simpler than those with coinfection with human mastadenovirus B. Prolonged fever days were associated with human mastadenovirus $B$ coinfection.
\end{abstract}

\section{Introduction}

M. pneumoniae is an important pathogen of communityacquired pneumonia in children $[1,2]$. Infection by M. pneumoniae is generally self-limited but can lead to severe pneumonia requiring intensive care [3]. It can also cause multiple extrapulmonary manifestations, involving the skin, musculoskeletal, nervous, hematological, digestive, and renal systems [4]. In addition, damage to the epithelial cells and cilia of the human airway can occur [5], affecting the function of the mucus-ciliary clearance system $[5,6]$ and host immunity [7], in turn increasing the rate of coinfection by opportunistic pathogens. The coinfection rate has been reported to reach $27 \%-48 \%$ in M. pneumoniae pneumonia (MPP) $[8,9]$ and are also closely associated with refractory MPP (RMPP) [10].

Human adenovirus (HAdV) is an important pathogen of the respiratory tract in children, accounting for $4-10 \%$ of pediatric community-acquired pneumonia [11]. M. pneumoniae, co-infected with HAdV in children, has been reported with more serious clinical manifestations [12]; however, the pathogenesis of coinfection of HAdV and M. pneumoniae has not been clarified. The microbiota has been reported to change during the lower respiratory tract 
(LRT) infection, and the change is closely related to the course or prognosis of pneumonia $[13,14]$. Nevertheless, it is currently unknown whether the microbiota in the LRT is associated with coinfection of HAdV and M. pneumoniae. We hypothesized that knowledge of the characteristics of the pediatric LRT microbiota in RMPP, coinfected with HAdV, may offer opportunities to uncover the mechanisms of pathogenesis of the coinfection, which is an unmet clinical need. Here, a case-control study was designed to achieve this goal.

\section{Methods}

2.1. Subjects and Groups. This is a prospective cohort study, in which the RMPP cases, hospitalized in the Children's Hospital of Fudan University from July 1, 2019, to June 30, 2020, were screened. RMPP is referred as clinical manifestations and the pulmonary images of $M$. pneumoniae pneumonia, showing deterioration after regular macrolide antibiotics treatment for more than seven days [15]. If signs of bronchial obstruction exist such as atelectasis or consolidation, bronchoscopy was performed. All RMPP cases received bronchoscopy, and informed consent form for bronchoscopy was obtained from the guardians. M. pneumoniae infection was confirmed by serological tests, positive for M. pneumoniae IgM or an IgG antibody titer $\geq 1: 160$ or with a $\geq 4$-fold increase (SeroMP ${ }^{\mathrm{TM}}$ IgM and SeroMP ${ }^{\mathrm{TM}}$ IgG test kit, Savyon Diagnostics Ltd) and by polymerase chain reaction showing $>2,500$ copies of $M$. pneumoniae genome per $\mathrm{mL}$ in the nasopharyngeal aspirate or BALF $(M$. pneumoniae nucleic acid amplification fluorescence detection kit, Daan Gene Co., Ltd., Guangzhou) [16]. HAdV infection was confirmed by a positive result of HAdV antigen from nasopharyngeal aspirates or BALF ( $\mathrm{D}^{3}$ Ultra DFA Respiratory Virus Screen \& ID kit, Diagnostic Hybrids, Inc). Patients with single M. pneumonia infection entered into the MP group, and those coinfected with $M$. pneumoniae and HAdV entered the MP\&ADV group. The exclusion criteria were (i) detection of any other pathogens in the patients' blood, nasopharyngeal aspirate, sputum, or BALF via culture, viral antigen detection assays, or serum tests and (ii) patients with chronic diseases, immune deficiencies, heart diseases, or using immunosuppressive drugs. The ethical application of this study was approved by the ethics committee of Children's Hospital of Fudan University on March 29, 2016 (No. 2016-87).

2.2. Clinical Characteristics and Laboratory Findings. Clinical information was collected, which included age, gender, and hospitalization days. The laboratory findings before bronchoscopy were also recorded, which comprised white blood cell (WBC) count, lymphocyte (LY) count, lymphocyte percentage (LY\%), neutrophil (Neu) count, neutrophil percentage $(\mathrm{Neu} \%), \mathrm{C}$-reactive protein (CRP), creatine kinase isoenzyme-MB (CK-MB), lactate dehydrogenase (LDH), levels of procalcitonin (PCT), D-dimer, alanine aminotransferase (ALT), aspartate aminotransferase (AST), lymphocyte subpopulations including percentages of $\mathrm{CD}^{+} \mathrm{T}$ cells, $\mathrm{CD}^{+} \mathrm{CD} 4^{+} \mathrm{T}$ cells,
$\mathrm{CD}^{+}{ }^{+} \mathrm{CD} 8^{+} \mathrm{T}$ cells, $\mathrm{CD} 16^{+} \mathrm{CD}^{2} 6^{+} \mathrm{T}$ cells, and $\mathrm{CD} 19^{+} \mathrm{T}$ cells, and lastly, humoral immunity that included immunoglobulin G (IgG), immunoglobulin M (IgM), immunoglobulin A $(\operatorname{IgA})$, and immunoglobulin E (IgE).

2.3. BALF Specimen Collection. Bronchoscopy was performed under conscious intravenous sedation with midazolam. Topical anesthesia of the larynx, trachea, and carina was achieved with $2 \%$ lidocaine (Sanchine, China), the bronchoscope was wedged in the lesion's segment or lobe, and the lavage was performed with three aliquots of sterile saline (Baxter, China), $1 \mathrm{ml} / \mathrm{kg}$ each, with a suction pressure of $100 \mathrm{~mm} \mathrm{Hg}$. All BALF samples were then immediately processed and stored according to the requirements of the laboratory.

2.4. DNA Extraction and Metagenomic Sequencing. DNA extraction from the BALF was performed as described in [17]. Briefly, $1 \mathrm{ml}$ BALF was digested with $50 \mu \mathrm{l}$ protease $\mathrm{K}$ at $60^{\circ} \mathrm{C}$ for $20 \mathrm{~min}$ and then placed at $4^{\circ} \mathrm{C}$ for $5 \mathrm{~min}$. The sample was transferred into a sterile $5 \mathrm{ml}$ tube, followed by brief centrifugation, and the DNA was extracted using the TIANamp Magnetic DNA kit (DP710-t2, Tiangen, China), according to the manufacturer's protocol. A no-template control (NTC) was performed for PCR. The quantity was assessed using the Qubit 2.0 fluorometer (Thermo Fisher Scientific, USA), and the quality of DNA was evaluated using the Nanodrop 8000 spectrophotometer (Thermo Fisher Scientific, USA). BALF DNA was fragmented into 150-300 bp size range by using the Bioruptor Pico Plus (Diagenode, Belgium) with the ultrasonication parameters as follows: $30 \mathrm{~s}$ on, $30 \mathrm{~s}$ off; 10 cycles. The DNA library was constructed using the KAPA HyperPrep kit (KAPA Biosystems, USA), according to the manufacturer's protocol. The library was qualified with Agilent 2100 (Agilent Technologies, CA) and sequenced on Illumina NextSeq 550Dx (Illumina, USA) using 75 bp single-ends.

2.5. Bioinformatics. Raw sequencing data were split by using bcl2fastq2, and clean reads were screened using Trimmomatic by removing low-quality reads, adapter contamination, duplications, and short (length $<35 \mathrm{bp}$ ) reads [17]. Bowtie 2 was then used to align with the human genome, and the unaligned sequences were retained. Kraken2 was used to identify the species contained in the sample, and Bracken was used to predict the actual relative abundance of the species in the sample.

2.6. Statistical Analysis. The statistical analyses were performed using the SPSS software (IBM, version 25.0); $P<0.05$ was defined as statistically significant. Other statistical analyses were performed using the R software (v4.0.1). Alpha diversity was measured using the Shannon index and Simpson index. Beta diversity was evaluated using the Bray-Curtis measure, compared by using the Wilcoxon rank sum test between the MP\&ADV and the MP groups, and visualized with the principal coordinate analysis (PCoA) 
TABLE 1: Demographical characteristics.

\begin{tabular}{lccc}
\hline Variable & MP $(n=21)$ & $\begin{array}{c}\text { MP\&ADV } \\
(n=11)\end{array}$ & $\begin{array}{c}P \\
\text { value }\end{array}$ \\
\hline Male/Female & $11 / 10$ & $7 / 4$ & 0.54 \\
Age (months) & $71 \pm 38$ & $59 \pm 18$ & 0.24 \\
Inpatient days (days) & $10.38 \pm 3.04$ & $10.70 \pm 5.25$ & 0.86 \\
Pleural effusion & $9(42.8 \%)$ & $3(27.3 \%)$ & 0.46 \\
Fever days & $11.05 \pm 3.10$ & $14.56 \pm 4.12$ & 0.04 \\
Extrapulmonary & $13(61.9 \%)$ & $5(45.5 \%)$ & 0.37 \\
manifestation & & & \\
Methylprednisolone & 21 & 11 & 1.0 \\
treatment & 8 & 7 & 0.13 \\
IVIG treatment & & &
\end{tabular}

TABLE 2: Laboratory findings.

\begin{tabular}{|c|c|c|c|}
\hline Variable & $\mathrm{MP}(n=21)$ & $\begin{array}{l}\text { MP\&ADV } \\
(n=11)\end{array}$ & $\begin{array}{c}P \\
\text { value }\end{array}$ \\
\hline $\begin{array}{l}\text { WBC counts }\left(\times 10^{9} /\right. \\
\text { L) }\end{array}$ & $12.61 \pm 4.98$ & $10.61 \pm 6.22$ & 0.34 \\
\hline NEU (\%) & $72.35 \pm 16.06$ & $66.30 \pm 20.57$ & 0.09 \\
\hline LY (\%) & $17.59 \pm 9.01$ & $25.28 \pm 18.83$ & 0.13 \\
\hline CRP & $52.10 \pm 48.37$ & $32.13 \pm 31.25$ & 0.19 \\
\hline CK-MB & $28.81 \pm 16.23$ & $25.40 \pm 11.82$ & 0.54 \\
\hline PCT & $0.26 \pm 0.28$ & $0.66 \pm 0.72$ & 0.12 \\
\hline $\mathrm{LDH}(\mathrm{IU} / \mathrm{ml})$ & $662.14 \pm 338.77$ & $601.82 \pm 474.19$ & 0.68 \\
\hline D-dimer (mg/L) & $3.25 \pm 2.31$ & $2.04 \pm 1.07$ & 0.07 \\
\hline $\operatorname{ALT}(\mathrm{U} / \mathrm{L})$ & $54.40 \pm 50.92$ & $27.22 \pm 44.29$ & 0.14 \\
\hline AST (U/L) & $51.03 \pm 44.36$ & $52.74 \pm 43.58$ & 0.92 \\
\hline \multicolumn{4}{|l|}{ Cellular immunity } \\
\hline $\mathrm{CD}^{+} \mathrm{T}(\%)$ & $61.55 \pm 15.63$ & $57.66 \pm 13.63$ & 0.53 \\
\hline $\mathrm{CD}^{+}{ }^{+} \mathrm{CD} 4^{+} \mathrm{T}(\%)$ & $34.69 \pm 10.70$ & $29.37 \pm 7.95$ & 0.20 \\
\hline $\mathrm{CD}^{+} \mathrm{CD}^{+} \mathrm{T}(\%)$ & $23.77 \pm 8.67$ & $24.84 \pm 11.56$ & 0.79 \\
\hline $\mathrm{CD}^{+} / \mathrm{CD} 8^{+} \mathrm{T}(\%)$ & $1.67 \pm 0.64$ & $1.47 \pm 0.82$ & 0.50 \\
\hline $\mathrm{CD} 6^{+} \mathrm{CD} 56^{+} \mathrm{T}(\%)$ & $8.54 \pm 4.19$ & $15.34 \pm 10.67$ & 0.02 \\
\hline $\mathrm{CD} 9^{+} \mathrm{T}(\%)$ & $28.33 \pm 14.94$ & $25.30 \pm 11.96$ & 0.60 \\
\hline \multicolumn{4}{|l|}{ Humoral immunity } \\
\hline $\operatorname{IgG}(g / L)$ & $12.22 \pm 5.62$ & $11.81 \pm 3.08$ & 0.84 \\
\hline $\operatorname{IgA}(\mathrm{g} / \mathrm{L})$ & $1.48 \pm 0.51$ & $1.23 \pm 0.57$ & 0.25 \\
\hline $\operatorname{IgM}(\mathrm{g} / \mathrm{L})$ & $3.31 \pm 2.29$ & $3.45 \pm 2.06$ & 0.88 \\
\hline $\operatorname{IgE}(\mathrm{kU} / \mathrm{L})$ & $496.91 \pm 809.76$ & $383.34 \pm 325.92$ & 0.61 \\
\hline
\end{tabular}

plot. The "vegan" $\mathrm{R}$ package was used to perform permutational multivariate analysis of variance (PERMANOVA) to analyze the Bray-Curtis distance in the MP\&ADV and MP groups. The Kruskal-Wallis rank-sum test ( $\mathrm{R}$ package "kruskal.test") was used to test differential relative abundance of taxonomic groups at the genus level. The "cor.test" $\mathrm{R}$ package was used to assess Spearman's correlations between clinical characteristics and the relative abundances of the genera, and the FDR correction was used to adjust all $P$ values.

\section{Results}

3.1. Clinical Characteristics. In this study, 21 subjects, consisting of 11 males and 10 females, were entered into the MP group, and 11 subjects, consisting of 7 males and 4 females, were entered into the MP\&ADV group. All eleven patients in the MP\&ADV group were coinfected with human mastadenovirus species $B$. There was no significant difference in gender distribution, age, or inpatient days between two groups (Table 1). The fever days were significantly longer in the MP\&ADV group than those in the MP group $(P<0.05)$ (Table 1$)$. There were 13 patients $(61.9 \%)$ with extrapulmonary manifestation in the MP group, including 8 with myocardial damage, 4 patients with liver damage, and 1 with liver damage and urticaria. There were 5 patients $(45.5 \%)$ with extrapulmonary manifestation in the MP\&ADV group, including 4 with myocardial damage and 1 with liver damage. There was no significant difference in the extrapulmonary manifestation or in treatments with methylprednisolone or IVIG between the two groups $(P>0.05$, respectively) (Table 1$)$.

3.2. Laboratory Findings. In laboratory findings, there were no significant differences in the WBC count or levels of CRP, CK-MB, PCT, LDH, D-dimer, ALT, or AST between 2 groups $(P>0.05)$ (Table 2$)$. In cellular immunity, there were also no significant differences in the percentages of $\mathrm{CD}^{+}$, $\mathrm{CD}^{+} \mathrm{CD}^{+}, \mathrm{CD}^{+} \mathrm{CD}^{+}, \mathrm{CD}^{+} / \mathrm{CD}^{+}$, and $\mathrm{CD} 19^{+}$cells in lymphocyte subpopulations between 2 groups $(P>0.05)$, while the percentage of $\mathrm{CD} 16^{+} \mathrm{CD} 56^{+}$cells was significantly higher in the MP\&ADV group than those in the MP group $(P<0.05)$ (Table 2$)$. In humoral immunity, there was no significant difference in the levels of IgG, IgA, IgM, or IgE ( $P>0.05$, respectively) (Table 2$)$. It was worth mentioning that the levels of IgE in both groups were much higher than the normal range, which is less than $100 \mathrm{kU} / \mathrm{L}$.

3.3. $\alpha$-Diversity of the BALF Microbiome. The $\alpha$-diversity was determined using the Shannon and Simpson indexes, representing the richness and evenness of the microbiota, respectively. There were no significant differences in the $\alpha$-diversity between the MP and the MP\&ADV group (Figure 1).

3.4. $\beta$-Diversity of the BALF Microbiome. Principal coordinate analysis (PCoA) provided an overview of the BALF microbiome and reflected the $\beta$-diversities of the different groups. The $\beta$-diversity was clearly higher in the MP\&ADV group than that in the MP group (Figure 2(a)). In addition, there was a significant difference in the $\beta$-diversity based on the Bray-Curtis distance between the two groups $(P<0.001$, PERMANOVA), suggesting that the intragroup difference of the MP group was smaller than that of the MP\&ADV group (Figure 2(b)).

3.5. The Taxa between the MP and MP\&ADV Groups. The most abundant taxa between the two study groups were compared (Figure 3). The top phylum of the MP BALF microbiota was Tenericutes at the phylum level. In contrast, the top phylum of the MP\&ADV BALF microbiota was Preplasmiviricota. The relative abundance of the two phyla was also significantly different $(P<0.001)$. 


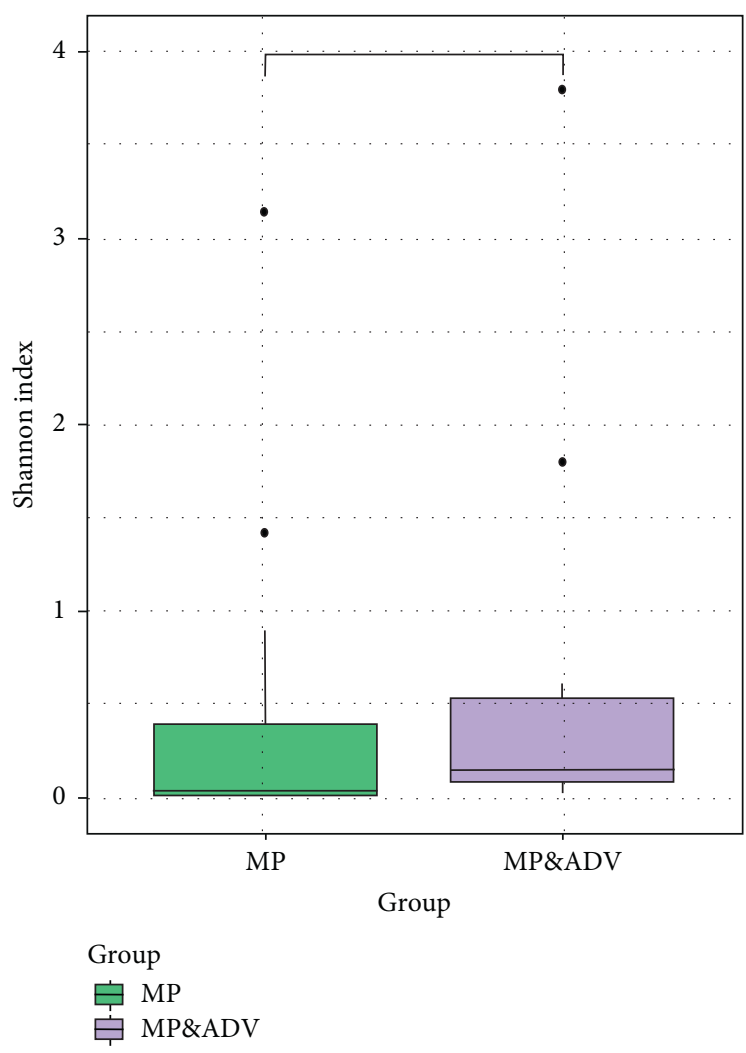

(a)

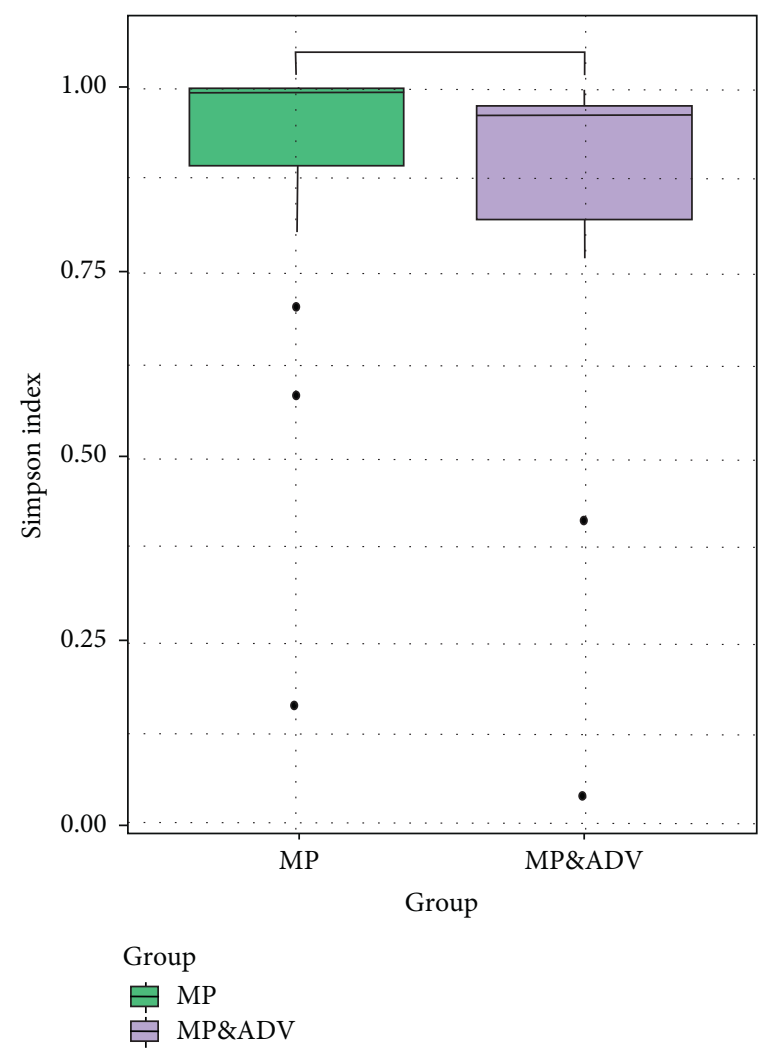

(b)

Figure 1: Comparison of the $\alpha$-diversity of microbial communities between the MP and MP\&ADV groups. Shannon's index (a) and Simpson index (b) showed no significant difference between the two groups.

3.6. Correlation between Microbiota and Clinical Measures. Spearman correlation analysis of clinical measures, such as age (months), fever days, inpatient days, CRP, WBC, Neu, Neu\%, LY, LY\%, PCT, D-dimer, LDH, CK-MB, ALT, AST, and immunological indices, and the most abundant 10 BALF microbiota species was performed. As shown (Figure 4), there was a strong positive correlation (correlation coefficient $=0.53, P<0.01$ ) between human mastadenovirus $B$ and fever days, whereas a strong negative correlation (correlation coefficient $=-0.50, P<0.01$ ) was observed between Mycoplasma pneumoniae and PCT. As far as immunological indices, there was a strong positive correlation (correlation coefficient $=0.45, \quad \mathrm{P}<0.01$ ) between $\mathrm{Myco}$ plasma pneumoniae and IgA level and a positive correlation (correlation coefficient $=0.36, P<0.05$ ) between human mastadenovirus $B$ and percentage of $\mathrm{CD} 16^{+} \mathrm{CD} 56^{+}$cells.

\section{Discussion}

In the current study, we found that patients coinfected with human mastadenovirus $B$ had a longer duration of fever than those with $M$. pneumoniae monoinfection, and the percentage of $\mathrm{CD} 6^{+} \mathrm{CD} 56^{+}$cells was also significantly higher in the coinfection group than those in the MP monoinfection group. In the microbiome study, we found that the BALF microbiome of $M$. pneumoniae monoinfection showed a decline in species richness compared with coinfection; however, the $\beta$-diversity was higher in the MP\&ADV group than that in the MP group. In the correlation between the microbiota and clinical measures, we found that human mastadenovirus $B$ was positively correlated with patients' fever days, and M. pneumonia was positively correlated with the level of IgA, whereas M. pneumoniae was negatively correlated with PCT.

M. pneumoniae damages the epithelial cells and cilia of the human airway, promoting mixed infections. The coinfection rate can reach $48 \%$ in MPP [9]. HAdV is an important pathogen of respiratory tract infection in children and is responsible for $4-10 \%$ of pediatric CAP [11]. Zhou et al. found that HAdV was the most prevalent coinfecting organism in M. pneumonia infection and was associated with RMPP [10]. In the current study of RMPP, we found that patients coinfected with human mastadenovirus $B$ had a longer fever duration compared with M. pneumoniae infection alone, which was consistent with the studies of Zhou et al. and Gao et al. [10,12], respectively, both of which showed longer fever in the MP\&ADV cohort. It seems reasonable that coinfection with $\mathrm{HAdV}$ prolongs the clearance time of the pathogen and affects the host immune response, leading to longer inflammation times. In terms of hospital days, we did not find any significant difference between the coinfection and the monoinfection groups. However, Gao's study showed longer hospital stays in coinfection with HAdV [12]. The apparent contradiction may be related to the study populations, which in Gao's study was just MPP patients, whereas they were RMPP patients in ours. 


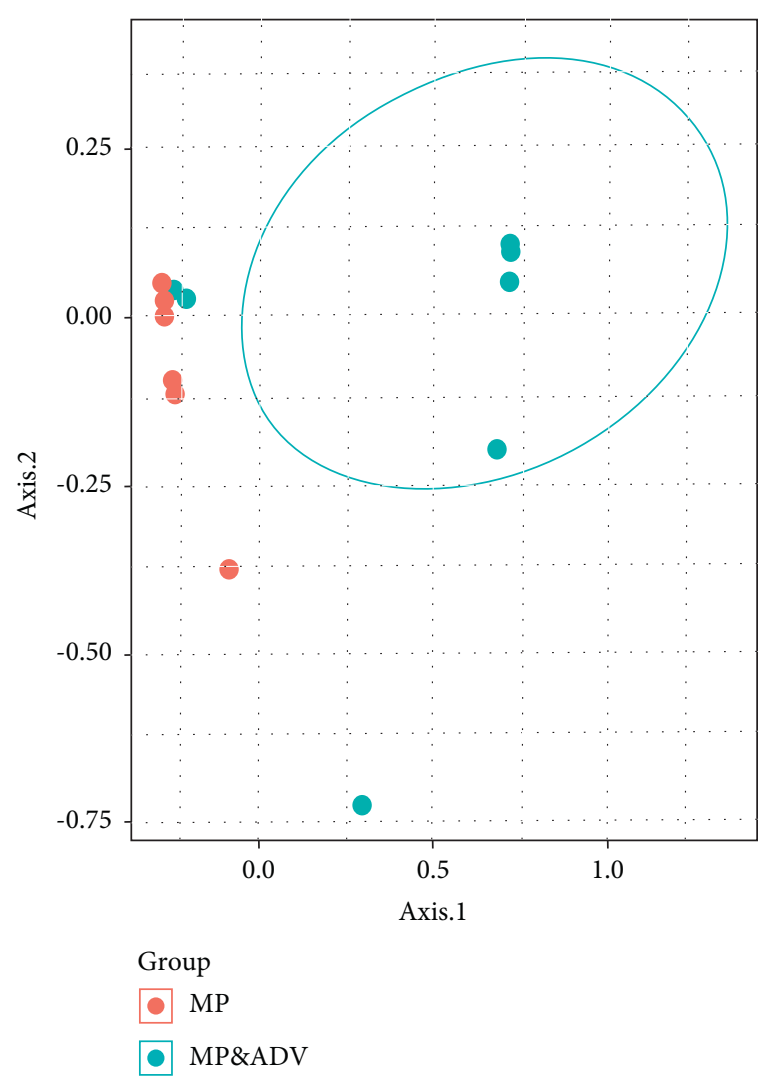

(a)

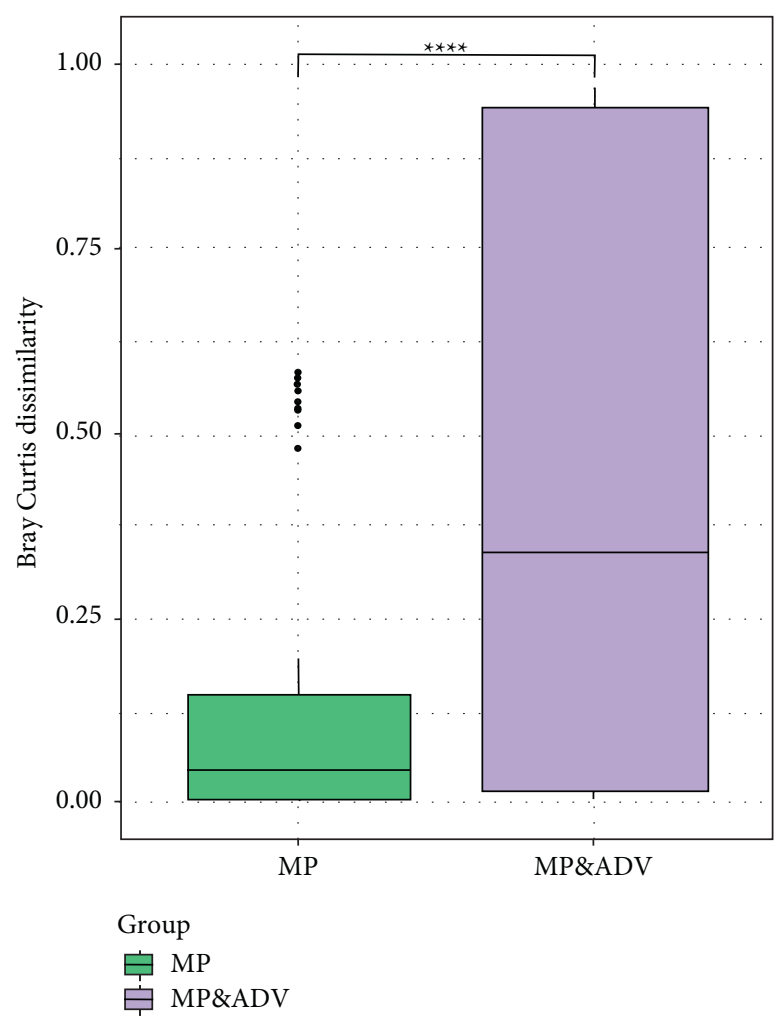

(b)

Figure 2: Comparison of the $\beta$-diversity of microbial communities between the MP and MP\&ADV groups. Each plot represents one community from one patient. (a) PCoA plot based on Bray-Curtis distance. Each plot represents one community from one patient. (b) $\beta$-diversity based on the Bray-Curtis distance of two groups. ${ }^{* * * *} P<0.001$. PERMANOVA, permutational multivariate analysis of variance.

In this study, we found that incidence of M. pneumoniaerelated extrapulmonary manifestations was around 50\% in both groups, and were mainly liver damage and myocardial damage. The incidence was higher than that in the reported data [18-20], which may be associated with the study's populations. As far as the immunological workup, there was no significant difference in the levels of IgG, $\operatorname{IgA}$, IgM, or IgE between the two groups. Some studies reported that serum IgE or atopy was associated with $M$. pneumoniae-related extrapulmonary manifestations [21, 22]. Another study showed that $\operatorname{IgE}$ was an independent risk factor for severe adenovirus pneumonia in children [23]. The results are not contradictory because the study populations are different. RMPP patients were our study populations, and the IgE level in our study was higher than that in the reported data $[21,22]$. Interestingly, Spearman correlation analysis showed that $M$. pneumoniae was positively correlated with the IgA level, suggesting that M. pneumoniae infection is associated with airway mucosal immunity. In addition, we found that the percentage of $\mathrm{CD} 16^{+} \mathrm{CD} 56^{+}$cells was significantly higher in the MP\&ADV group than that in the MP group. Spearman correlation analysis also showed that human mastadenovirus $B$ positively correlated with the percentage of $\mathrm{CD} 16+\mathrm{CD} 56+$ cells. $\mathrm{CD} 16^{+} \mathrm{CD} 56^{+}$cells are known as natural killer $(\mathrm{NK})$ cells in humans. NK cells are important mediators of antiviral innate immunity that can be activated by HAdV infection [24, 25].

In the current study, we recruited children of RMPP coinfected with HAdV to study the characteristics of the BALF microbiome composition in these patients in China. The BALF microbiome of $M$. pneumoniae monoinfection showed a decline in species richness compared with coinfection, but the difference was not significant. As regards the $\beta$-diversity, it was clearly higher in the MP\&ADV group than in the MP group in our study, suggesting that the intragroup difference of the MP group was smaller than that of the MP\&ADV group. In a study by Wang et al., the bacterial diversity in $M$. pneumoniae pneumonia was found to be lower than that in adenovirus pneumonia [26]. In terms of coinfection with bacteria, $M$. pneumoniae was found to compete for nutrients to eliminate other bacteria [27] and activate the host inflammatory response [28]. As a result, the bacterial diversity was small in MPP [29-31]. In terms of coinfection with the virus, virus infections impair the lung epithelial layer and suppress the immune response, which promotes bacterial outgrowth and frequently leads to secondary bacterial infections [32], and this may increase the bacterial diversity. Consistent with this theory, we also found that when coinfected with $\mathrm{HAdV}$, the bacterial diversity was increased. 


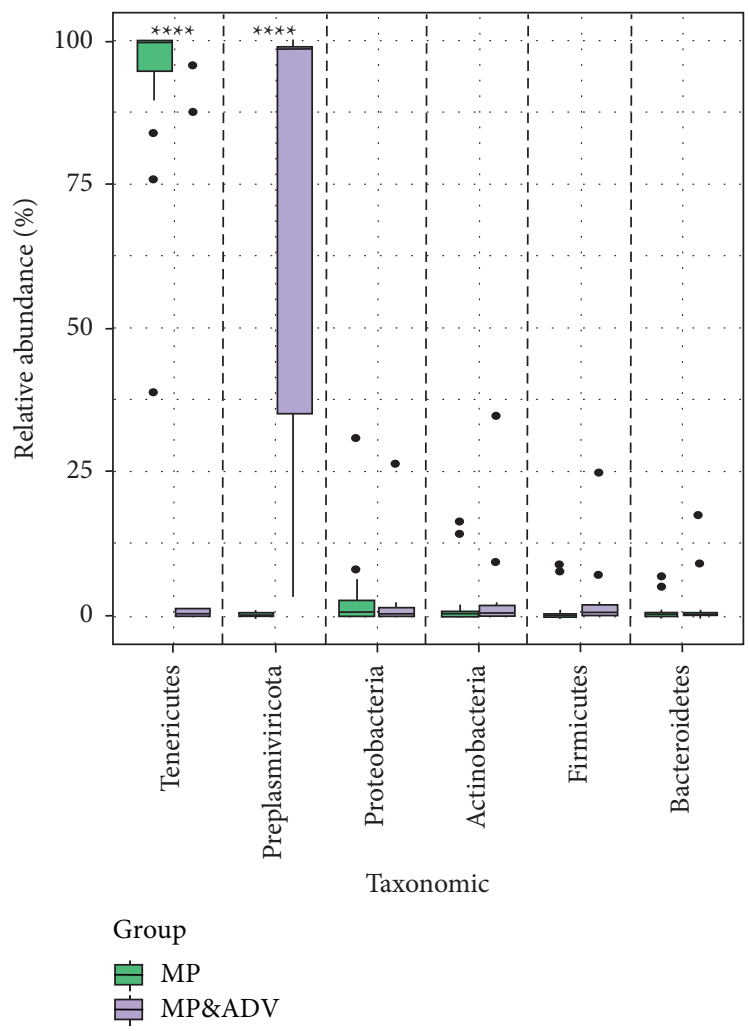

FIGURE 3: The most abundant phylum (mean relative abundances greater than $0.4 \%$ and penetrance greater than $40 \%$ among all samples) among the two groups. ${ }^{*} P<0.05,{ }^{* *} P<0.01$, and ${ }^{* * *} P<0.001$. Kruskal-Wallis rank-sum test.

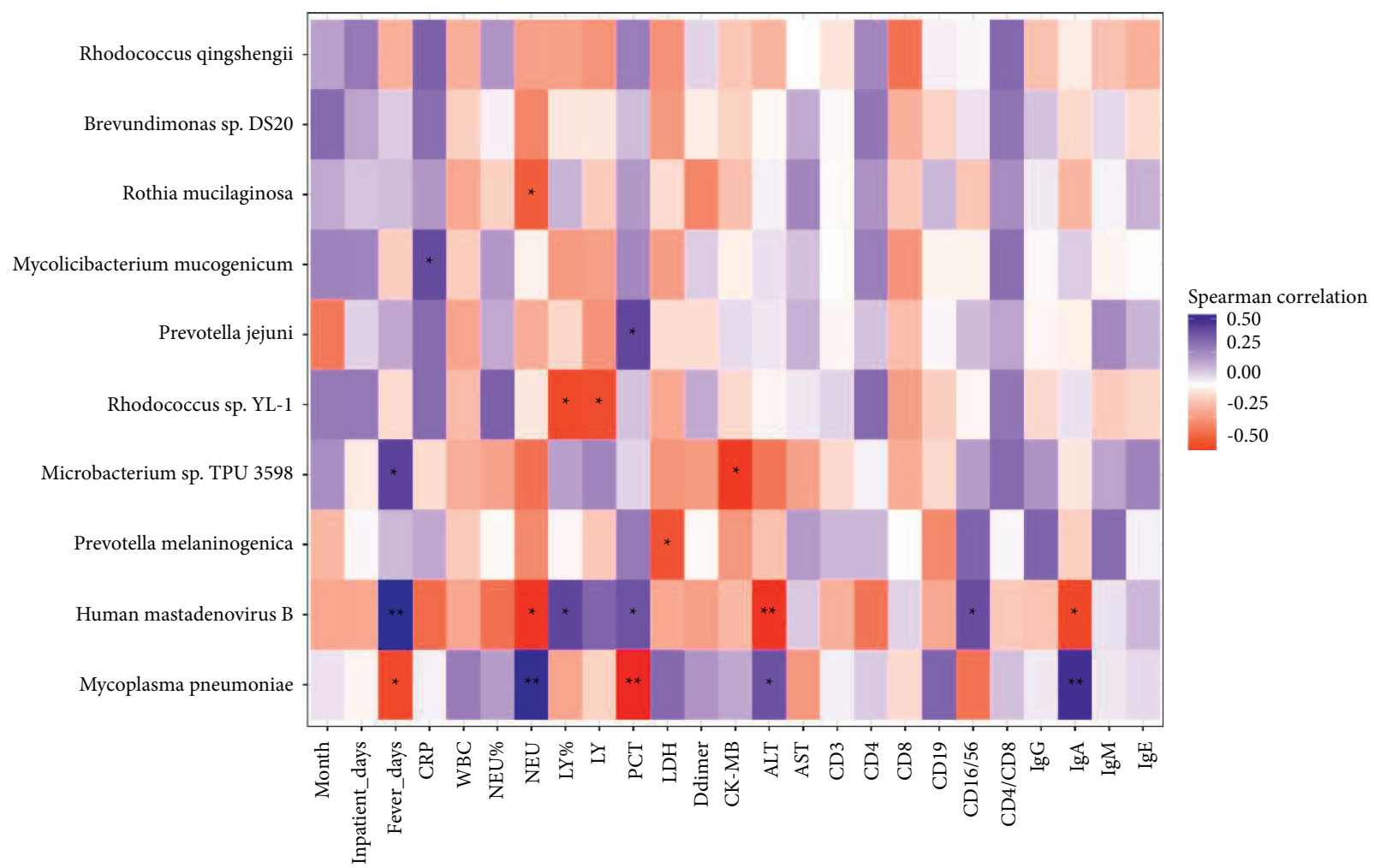

Figure 4: Heatmap of Spearman correlations between clinical measures and microbiota species. WBC, white blood cell counts; LY, lymphocyte count; LY\%, lymphocyte percentage; Neu, neutrophil count; Neu\%, neutrophil percentage; PCT, procalcitonin; CRP, C-reactive protein; $\mathrm{LDH}$, lactate dehydrogenase; CK-MB, creatine kinase isoenzyme-MB; ALT, alanine aminotransferase; AST, aspartate aminotransferase. ${ }^{*} P<0.05$ and ${ }^{* *} P<0.01$. 
Lastly, there were significant relationships between clinical measures and the microbiota, as shown by Spearman correlation analysis. Human mastadenovirus $B$ was positively correlated with patients' fever days, indicating that human mastadenovirus $B$ may contribute to symptoms. In addition, $M$. pneumoniae was negatively correlated with PCT. M. pneumoniae is a bacterial pathogen, and PCT is considered as a biomarker of infections. Our results suggest that the correlations of etiological diagnosis and biomarkers of infection remain unsolved problems.

We would like to mention several potential limitations of this study. First, the sample size was limited, especially in the MP\&ADV group. Second, our quantification of the bacteria was only at the DNA level, which cannot distinguish live and dead microorganisms and cannot detect RNA viral genomes. Third, the study population in this study was RMPP patients, which is very limited.

In conclusion, this study showed that the microbiota of the BALF in children with RMPP of M. pneumoniae monoinfection was much simpler than those with coinfection with human mastadenovirus $B$. The coinfection was also associated with prolonged fever duration. These results contribute important profiles of the lung microbiota and fill a gap in our knowledge of the mechanism of pathogenesis in RMPP, coinfected with human mastadenovirus $B$.

\section{Data Availability}

All the data included in this study are available upon request to the corresponding author.

\section{Conflicts of Interest}

The authors declare that they have no conflicts of interest.

\section{Authors' Contributions}

Dr Aizhen Lu conceptualized and designed the study and reviewed the manuscript. Dr Libo Wang reviewed the protocol and the manuscript; Drs Zhimin Xi, Yanyan Shi, and Lijuan Liu participated in the trial selection and data collection. Jinglong Chen participated in data analysis and interpretation. Dr Wenxiang Zhou participated in data analysis and wrote the manuscript. All the authors approved the manuscript.

\section{Acknowledgments}

The authors gratefully acknowledge the statistical and bioinformatics help of Dinfectome Technology Inc. (https:// www.dinfectome.com/). This work was supported by the National Natural Science Foundation of China (Grant no. 81601332).

\section{References}

[1] L. E. Rogozinski, B. K. Alverson, and E. A. Biondi, "Diagnosis and treatment of Mycoplasma pneumoniae in children," Minerva Pediatrica, vol. 69, no. 2, pp. 156-160, 2017.
[2] A. K. C. Leung, A. H. C. Wong, and K. L. Hon, "Communityacquired pneumonia in children," Recent Patents on Inflammation \& Allergy Drug Discovery, vol. 12, no. 2, pp. 136-144, 2018.

[3] K.-L. Lee, C.-M. Lee, T.-L. Yang et al., “Severe Mycoplasma pneumoniae pneumonia requiring intensive care in children, 2010-2019," Journal of the Formosan Medical Association, vol. 120, no. 1, pp. 281-291, 2021.

[4] D. Poddighe, "Extra-pulmonary diseases related to Mycoplasma pneumoniae in children: recent insights into the pathogenesis," Current Opinion in Rheumatology, vol. 30, no. 4, pp. 380-387, 2018.

[5] K. B. Waites and D. F. Talkington, "Mycoplasma pneumoniae and its role as a human pathogen," Clinical Microbiology Reviews, vol. 17, no. 4, pp. 697-728, 2004.

[6] Y. Hao, Z. Kuang, J. Jing et al., "Mycoplasma pneumoniae modulates STAT3-STAT6/EGFR-FOXA2 signaling to induce overexpression of airway mucins," Infection and Immunity, vol. 82, no. 12, pp. 5246-5255, 2014.

[7] J. L. Medina, J. J. Coalson, E. G. Brooks et al., "Mycoplasma pneumoniae CARDS toxin induces pulmonary eosinophilic and lymphocytic inflammation," American Journal of Respiratory Cell and Molecular Biology, vol. 46, no. 6, pp. 815-822, 2012.

[8] X. Zhang, Z. Chen, W. Gu et al., "Viral and bacterial coinfection in hospitalised children with refractory Mycoplasma pneumoniae pneumonia," Epidemiology and Infection, vol. 146, no. 11, pp. 1384-1388, 2018.

[9] I. C. Michelow, K. Olsen, J. Lozano et al., "Epidemiology and clinical characteristics of community-acquired pneumonia in hospitalized children," Pediatrics, vol. 113, no. 4, pp. 701-707, 2004.

[10] Y. Zhou, J. Wang, W. Chen et al., "Impact of viral coinfection and macrolide-resistant mycoplasma infection in children with refractory Mycoplasma pneumoniae pneumonia," $B M C$ Infectious Diseases, vol. 20, no. 1, p. 633, 2020.

[11] J. D. C. Adenoviruses, Textbook of Pediatric Infectious Diseases, R. Feigin, Ed., pp. 1666-84, WB Saunders, Philadelphia, 1998.

[12] J. Gao, L. Xu, B. Xu, Z. Xie, and K. Shen, "Human adenovirus Coinfection aggravates the severity of Mycoplasma pneumoniae pneumonia in children," BMC Infectious Diseases, vol. 20, no. 1, p. $420,2020$.

[13] C. Thibeault, N. Suttorp, and B. Opitz, "The microbiota in pneumonia: from protection to predisposition," Science Translational Medicine, vol. 13, no. 576, p. 13, Article ID eaba0501, 2021.

[14] S. Hanada, M. Pirzadeh, K. Y. Carver, and J. C. Deng, "Respiratory viral infection-induced microbiome alterations and secondary bacterial pneumonia," Frontiers in Immunology, vol. 9, Article ID 2640, 2018.

[15] K.-Y. Lee, H.-S. Lee, J.-H. Hong et al., "Role of prednisolone treatment in severe Mycoplasma pneumonia pneumonia in children," Pediatric Pulmonology, vol. 41, no. 3, pp. 263-268, 2006.

[16] W. J. Lee, E. Y. Huang, C. M. Tsai et al., "Role of serum mycoplasma pneumoniae IgA, IgM, and IgG in the diagnosis of mycoplasma pneumoniae-related pneumonia in school-age children and adolescents," Clinical and Vaccine Immunology: CVI, vol. 24, pp. e00471-16, 2017.

[17] J. Chen, Z. Xi, Y. Shi et al., "Highly homogeneous microbial communities dominated by Mycoplasma pneumoniae instead of increased resistance to macrolide antibiotics is the characteristic of lower respiratory tract microbiome of children 
with refractory Mycoplasma pneumoniae pneumonia," Translational Pediatrics, vol. 10, no. 3, pp. 604-615, 2021.

[18] Expert Committee on Rational use of Medicines for Children Pharmaceutical Group National Health and Family Planning Commission, "Expert consensus on laboratory diagnostics and clinical practice of mycoplasma pneumoniae infection in children in China (2019)," Zhonghua Er Ke Za Zhi, vol. 58, no. 5, pp. 366-373, 2020, Chinese.

[19] D. Poddighe, "Mycoplasma pneumoniae-related hepatitis in children," Microbial Pathogenesis, vol. 139, Article ID 103863, 2020.

[20] A. Paz and I. Potasman, "Mycoplasma-associated carditis. Case reports and review," Cardiology, vol. 97, no. 2, pp. 83-88, 2002.

[21] D. Poddighe, E. V. Comi, I. Brambilla, A. Licari, P. Bruni, and G. L. Marseglia, "Increased total serum immunoglobulin E in children developing mycoplasma pneumoniae-related extrapulmonary diseases," Iranian Journal of Allergy, Asthma, and Immunology, vol. 17, no. 5, pp. 490-496, 2018.

[22] Z. Wang, J. Sun, Y. Liu, and Y. Wang, "Impact of atopy on the severity and extrapulmonary manifestations of childhood Mycoplasma pneumoniae pneumonia," Journal of Clinical Laboratory Analysis, vol. 33, no. 5, Article ID e22887, 2019.

[23] H. Zhong and X. Dong, "Analysis of clinical characteristics and risk factors of severe adenovirus pneumonia in children," Frontiers in Pediatrics, vol. 9, Article ID 566797, 2021.

[24] J. M. Routes, "Measurement of natural-killer cell lytic activity of adenovirus-infected or adenovirus-transformed cells," Methods in Molecular Medicine, vol. 131, pp. 213-219, 2007.

[25] J. H. W. Pahl, D. H. J. Verhoeven, K. M. C. Kwappenberg et al., "Adenovirus type 35, but not type 5, stimulates NK cell activation via plasmacytoid dendritic cells and TLR9 signaling," Molecular Immunology, vol. 51, no. 1, pp. 91-100, 2012.

[26] H. Wang, Q. Zhou, W. Dai et al., "Lung microbiota and pulmonary inflammatory cytokines expression vary in children with tracheomalacia and adenoviral or mycoplasma pneumoniae pneumonia," Frontiers in Pediatrics, vol. 7, Article ID 265, 2019.

[27] J. Yang, W. C. Hooper, D. J. Phillips, and D. F. Talkington, "Cytokines in Mycoplasma pneumoniae infections," Cytokine \& Growth Factor Reviews, vol. 15, no. 2-3, pp. 157-168, 2004.

[28] C. Peteranderl, J. I. Sznajder, S. Herold, and E. Lecuona, "Inflammatory responses regulating alveolar ion transport during pulmonary infections," Frontiers in Immunology, vol. 8, Article ID 446, 2017.

[29] S. M. P. J. Prevaes, W. A. A. de Steenhuijsen Piters, K. M. de Winter-de Groot et al., "Concordance between upper and lower airway microbiota in infants with cystic fibrosis," European Respiratory Journal, vol. 49, no. 3, Article ID 1602235, 2017.

[30] S. I. Miller, R. K. Ernst, and M. W. Bader, "LPS, TLR4 and infectious disease diversity," Nature Reviews Microbiology, vol. 3, no. 1, pp. 36-46, 2005.

[31] F. Zhao, G. Liu, J. Wu et al., "Surveillance of macrolide-resistant mycoplasma pneumoniae in beijing, China, from 2008 to 2012," Antimicrobial Agents and Chemotherapy, vol. 57, no. 3, pp. 1521-1523, 2013.

[32] J. C. Brealey, P. D. Sly, P. R. Young, and K. J. Chappell, "Viral bacterial co-infection of the respiratory tract during early childhood," FEMS Microbiology Letters, vol. 362, Article ID fnv062, 2015. 